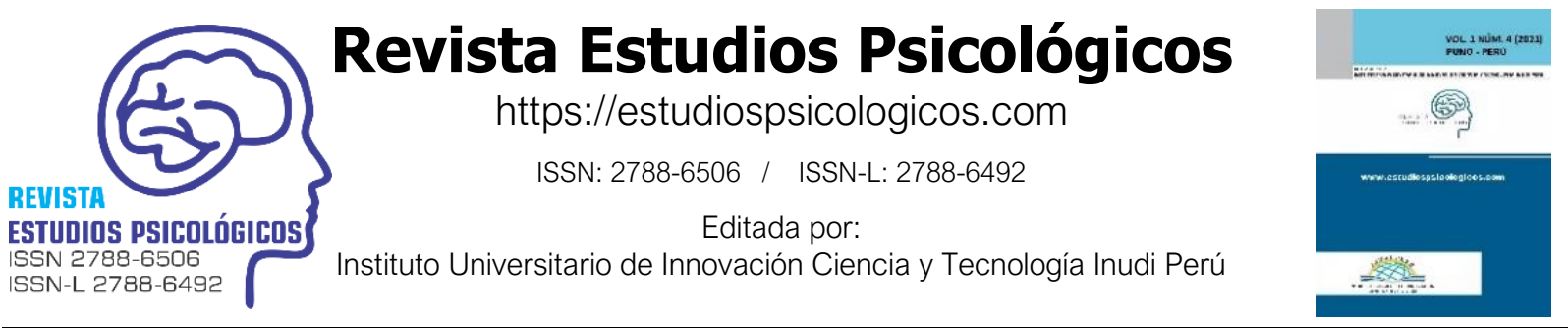

ARTÍCULO DE REVISIÓN

\title{
Prácticas de atención psicosocial para docentes orientadores y convivencia escolar en educación básica secundaria: una revisión sistemática
}

\author{
Psychosocial care practices for guidance teachers and school coexistence in basic secondary \\ education: a systematic review
}

Práticas de atenção psicossocial para orientadores e convivência escolar no ensino médio: uma revisão sistemática

\section{Sunilda Soto ${ }^{1}$}

Universidad Simón Bolívar, Barranquilla, Colombia

(iD) https://orcid.org/0000-0001-6202-5068

\author{
Lizeth Reyes \\ Universidad Simón Bolívar, Barranquilla, Colombia \\ (iD) https://orcid.org/0000-0002-9469-8387
}

D0I: https://doi.org/10.35622/j.rep.2022.01.008

Recibido 11/12/2021/ Aceptado 11/01/2022

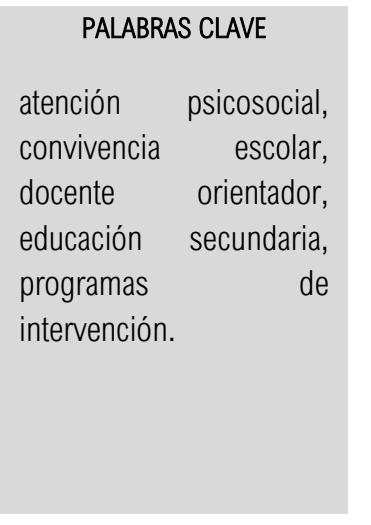

KEYWORDS

psychosocial care, school coexistence, guidance teacher, secondary

\begin{abstract}
RESUMEN. La convivencia escolar ha sido un fenómeno enfocado en abordar la violencia dentro de la escuela. La presente revisión sistemática tiene como objetivo identificar y describir los programas de intervención de convivencia escolar. Se utilizó el protocolo de revisión sistemática Prisma, bajo una búsqueda temática en los tesauros de base d datos confiables. Como criterio de inclusión las publicaciones tenían que estar relacionadas con los programas de convivencia y los factores que influyen en la convivencia escolar en educación básica secundaria entre 2012 y 2021. El número de registros identificados en la búsqueda fue 251 documentos. Se recuperó el artículo completo y se examinó cuidadosamente su contenido, obteniendo 50 artículos elegibles para su revisión bajo criterios de inclusión un total de 19 artículos incluidos. Los estudios encontrados hacen énfasis en la necesidad de seguir promoviendo la construcción programas de intervención y formación escolar, basados en los planes de convivencia. Se apuesta por la necesidad de crear un modelo nuevo de convivencia que supere los retos de la educación actual.
\end{abstract}

ABSTRACT. School coexistence has been a phenomenon focused on addressing violence within the school. The present systematic review aims to identify and describe the intervention programs for school coexistence. The Prisma systematic review protocol was used under a thematic search in reliable database thesauri. As inclusion criteria, publications related to coexistence programs and the factors influencing school coexistence in primary-secondary education between 2012 and 2021. The number of records identified in the search was 251 documents. The full article was retrieved,

\footnotetext{
${ }^{1}$ Correspondencia: sunilda.soto@unisimon.edu.co
} 


\begin{tabular}{lll}
\hline $\begin{array}{l}\text { education, intervention } \\
\text { programs. }\end{array}$ & $\begin{array}{l}\text { and its content was carefully examined, obtaining } 50 \text { pieces eligible for review under inclusion } \\
\text { criteria, a total of } 19 \text { pieces included. The studies emphasized the need to continue promoting } \\
\text { school intervention and training programs based on coexistence plans. It is committed to the need } \\
\text { to create a new model of coexistence that overcomes the challenges of current education. }\end{array}$ \\
\hline
\end{tabular}

PALAVRAS-CHAVE
atenção psicossocial,
convivência escolar,
orientação de professor,
ensino médio, programas
de intervenção.

RESUMO. A convivência escolar tem sido um fenômeno voltado para o enfrentamento da violência
dentro da escola. A presente revisão sistemática tem como objetivo identificar e descrever os
programas de intervenção para a convivência escolar. Foi utilizado o protocolo de revisão
sistemática Prisma, por meio de uma busca temática em tesauros de bases de dados confiáveis.
Como critério de inclusão, as publicaçães deveriam estar relacionadas aos programas de
convivência e aos fatores que influenciam a convivência escolar no ensino médio entre 2012 e
2021. 0 número de registros identificados na busca foi de 251 documentos. 0 artigo completo foi
recuperado e seu conteúdo foi criteriosamente examinado, obtendo-se 50 artigos elegíveis para
revisão sob os critérios de inclusão, totalizando 19 artigos incluídos. Os estudos encontrados
reforçam a necessidade de continuar a promover a construção de programas de intervenção e
formação escolar, com base em planos de convivência. Está comprometida com a necessidade de
criar um novo modelo de convivência que supere os desafios da educação atual.

\section{INTRODUCCIÓN}

La convivencia escolar es un fenómeno de interés para la comunidad científico-educativa sobre la necesidad de una educación basada en valores de convivencia y una educación preventiva de conductas antisociales (Penalva et al. 2013). Es decir, junto con la investigación se crea la necesidad de proponer una educación social y democrática en las instituciones educativas que prolifere la creación de programas de convivencia para afrontar las dificultades de interacción escolar (Basto et al. 2018). Según los enfoques de convivencia escolar para Latinoamérica, se encuentra que Colombia se orienta por la visión democrática, es decir, enfocada en los derechos humanos (Benítez, 2020). En función de ello se desarrolla la pedagogía más allá de la interacción docente-estudiante, que establece la articulación entre el Ministerio de Educación, el Ministerio de Salud, la Escuela y la Familia (Benítez, 2020; Cabrales et al. 2017). Los resultados de esta visión democrática- pedagógica, proponen la construcción de nuevas políticas públicas que resalten la necesidad de involucrar a la familia para contrarrestar la aparición de la violencia escolar (Roncancio et al. 2017). Asimismo, permite fortalecer las capacidades al interior de las instituciones educativas, en especial en los niveles de atención y seguimiento a la convivencia.

En aras de contribuir en la formación escolar y en mejorar la convivencia en las escuelas, se expidió la ley 1620 del 2013, como marco jurídico para respaldar los procesos. La Ley 1620 se encarga de crear el —Sistema Nacional de Convivencia Escolar y formación para el ejercicio de los derechos humanos, la educación para la sexualidad y la prevención y mitigación de la violencia escolar. La citada Ley aportó herramientas a la formación de ciudadanos activos para construir una sociedad democrática, participativa, pluralista e intercultural. 
En este orden de ideas, la presente revisión sistemática muestra el estado actual de la investigación acerca de la convivencia escolar en población estudiantil de básica secundaria los factores asociados a la convivencia escolar y la labor del docente orientador para abordar esta problemática. Como objetivo se buscó identificar y describir los programas de intervención de convivencia escolar para identificación de elementos que se deben tener presente para la atención psicosocial. Se formularon las siguientes preguntas que serán discutidas en la sección de discusión de resultados: ¿Cuáles son los programas de intervención para la convivencia escolar en población estudiantil de básica secundaria? ¿Cuál es la labor del docente orientador para abordar esta problemática? ¿Cuáles son los factores asociados en la convivencia escolar?

\section{METODO Y MATERIALES}

La revisión sistemática actualiza a los lectores respecto de temáticas específicas (Franco et al., 2018). En este artículo se utiliza el sistema Preferred Reporting Items for Systematic Reviews and Meta-Analyses (PRISMA) cuyo de reporte gráfico se especifica en la Figura 1 (Moher, et al., y The PRISMA Group, 2009). Bajo protocolo Prisma, se asume como ruta metodológica una búsqueda temática en los tesauros de MeSH, Dialnet, Redalyc, Scopus y Science Direct. Las siguientes ecuaciones definieron la búsqueda: (school coexistence) [Términos MeSH] OR (coexistence difficulties) AND (intervention programs) [Términos MeSH] AND (guidance teachers) AND (student interaction) OR (high school). Se establecieron los siguientes criterios:

a. Criterios de inclusión: las publicaciones tenían que estar relacionadas con los programas de convivencia y los factores asociados a la convivencia escolar en educación básica secundaria entre 2012 y 2021.

b. Criterios de exclusión: Documentos relacionados con educación básica primaria y revisiones sistemáticas o documentales.

La evaluación crítica de los artículos consistió en la lectura completa del estudio. Aquellos estudios publicados por duplicado o encontrados en más de una base de datos fueron considerados sólo una vez. La figura 1 muestra el diagrama del proceso de selección de estudios con mayor claridad. Primero, la búsqueda se dirigió a artículos de revistas publicados entre 2012-2021. Esta búsqueda preliminar reveló un total de 251 documentos. En segundo lugar, una selección de título fue realizado conteniendo las combinaciones antes mencionadas de términos de búsqueda en el título, el resumen o las palabras clave. Títulos no relacionados con el tema fueron descartados. Con respecto a la inclusión del resumen, se recuperó el artículo completo y se examinó cuidadosamente su contenido, obteniendo 50 artículos elegibles para su revisión. Posteriormente, se 
eliminaron además los títulos duplicados (29), obteniendo bajo criterios de inclusión y exclusión un total de 21 documentos incluidos en la revisión.

Se enumeraron los objetivos o preguntas de investigación planteados por los autores para determinar el alcance de los intereses de los investigadores. Se identificaron todos los métodos de recopilación y análisis de datos, los resultados y la población. En la tabla 1 se presentan los resultados teniendo en cuenta las características de los estudios. Finalmente, se presenta un resumen de los hallazgos principales, considerando su relevancia.

El tipo de estudio que prevaleció fue la investigación descriptiva (60\%), el estudio de caso (10\%) y las revisiones documentales (30\%). Los instrumentos más usados por los autores fueron: la triangulación de: entrevistas, encuestas, grupos focales, grupos de discusión, cuestionarios para la construcción de normas dentro del aula (70\%); pruebas para valorar el clima escolar, los cuestionarios de convivencia y de relaciones interpersonales (10\%); los métodos interrogativos (10\%); análisis de contenido (5\%) y el modelo de ecuaciones estructurales $(5 \%)$. 
Figura1 Selección de estudios: diagrama



Nota: $\left.{ }^{*}\right)$ Los artículos se excluyeron porque no mencionaban programas de intervención y no evaluaban población de estudiantes de secundaria. 


\section{RESULTADOS}

Se encontró que un total de 21 artículos eran relevantes para las combinaciones de búsqueda predefinidos y los criterios de inclusión, todos publicados entre 2012 y 2021. El tema de la convivencia escolar en población secundaria se ha investigado principalmente en España (50\%), en Alicante, Sevilla, Madrid, Córdoba, Murcia, Ciudad Real, la Mancha, Cáceres y Badajoz. En Colombia (30\%) en Bogotá, Barranquilla, Pereira, Boyacá, Pasto Nariño; en Chile (Coquimbo, la serena, y Maule 10\%) Venezuela (Mérida 5\%) y México (Ciudad Obregón 5\%). Los principales temas de interés fueron: Programas de intervención escolar para mejorar la convivencia escolar (70\%), factores asociados en la aparición de conductas que afectan la convivencia (15\%), el rol del docente orientador (10\%), aunado al papel de la familia (5\%).

La tabla 1 presenta las características de los estudios 


\section{Tabla 1. Características de los estudios}

País $\quad$ Referencias

Tipo de estudio

Participantes

Instrumentos

Resultados

$\begin{array}{lll}\text { Sevilla, } & \text { Jiménez, A. (2017). Estudio de caso } & \begin{array}{l}4 \text { alumnos } \\ \text { 4docentes } \\ \text { orientadores }\end{array} \quad \text { Grupos de autoayuda }\end{array}$

$\begin{array}{lll}33 & \text { profesores } & \text { Procedimiento descriptivo- } \\ \text { de } & \text { educación } & \text { interpretativo }\end{array}$

Los grupos de apoyo mutuo proporcionan una visión compartida de los problemas abriendo vías para iniciar diálogos en las escuelas que empoderen los propios recursos de los alumnos. Como consecuencia, mejora el ambiente y clima de los centros.

Entre los problemas de convivencia que más dificultan a los docentes: la falta de atención y el tiempo perdido en la disciplina.

Madrid, $\quad$ Carpio, C., y Tejero, Estudio de caso $\mathrm{J}(2012)$.

secundaria

programas basados en el diálogo. Se hace
necesario, promover la reflexión del docente.

La convivencia escolar afecta la labor docente, ellos son poco conscientes de su $\begin{array}{ll}33 & \text { profesores } \\ \text { de } & \text { educación }\end{array}$ Procedimiento descriptivo- $\begin{aligned} & \text { papel en el buen clima escolar. Se hace } \\ & \text { necesario promover la reflexión del docente }\end{aligned}$ Alicante, $\quad$ García, M. Sola, J., $\quad$ Peiró-i-Gregori, S. Estudio de caso España (2016). secundaria interpretativo para facilitar la formación del profesorado respecto a su incidencia en la resolución de conflictos. 
Roncancio, M.,

Bogotá,

Colombia

Camacho, N.,

Constanza, J., Vaca, Hermenéutico

P. (2017).

interpretativo

España

Cáceres, y

Macias, E. (2017).

Observacional

Nail, O., Muñoz, M., y Gajardo, J. (2013).

No experimental

Análisis descriptivo

No experimental desde la teoría de

Bogotá,

Colombia
Delgado, A, (2018). las representaciones sociales, para indagar la orientación escolar y la convivencia.
5 docentes

15 estudiantes

15 padres de

familia

Triangulación a partir de los planteamientos de la Teoría

Fundamentada propuesta

por Strauss \& Corbin (2002).

\section{Triangulación}

instrumentos

No menciona

investigación

(videograbación, entrevista y

cuestionario)

5 escuelas de secundaria de la provincia de Concepción, Chile.

Pauta de registro disrupción

Cuestionario para construcción democrática de normas en el aula

Familia vs escuela, rol del adulto y la convivencia en la escuela, dan cuenta de la influencia de la inteligencia emocional en la convivencia escolar. Se concluye avanzar en la interacción familia - escuela y orientar la construcción de propuestas educativas que aborden con sentido pedagógico la alfabetización emocional.

Las estrategias no verbales son invisibles a de (docente en el aula. Para una mayor eficacia de las actuaciones docentes, se concentran en el comportamiento de los profesores en el aula.

Alta gravedad a los problemas de convivencia en el aula: necesidad de la de construcción de las normas para mejorar la vida en común en el aula. Se procedió a

establecer círculos de conversación con el profesorado para la mejora en la convivencia y la construcción de normas.

Se evidenciaron cinco tipos de representaciones sociales: orientación 38 estudiantes, Métodos interrogativos, docentes y específicamente la encuesta directivos de semiestructurada y la secundaria. entrevista escolar como guía, acompañamiento, detección preventiva de aspectos convivenciales, intervención de problemas personales, familiares y sociales y como apoyo. Se destaca el rol del educador y se 
Murcia, Penalva, A.,

España

Hernández, M.,

Guerrero, C. (2013).

Panel de expertos

30 expertos

Panel de expertos como técnica metodológica

reafirma como un componente crucial: el afecto, donde está inmerso el diálogo, la escucha y el acompañamiento.

El 25\% de los expertos entienden que los conceptos de convivencia ayudarían al docente a realizar una gestión eficaz y el $16,16 \%$ consideran incluir las funciones del tutor. Se concede relevancia a las medidas educativas del docente (91,66\%). Las causas se centran en los factores escolares $(91,66 \%)$ y familiares $(66,66 \%)$.

Propuesta de convivencia escolar para Estudiantes $y$ padres de Revisión documental fomentar la paz en el contexto educativo, en

$\begin{array}{lll}\text { Boyacá, } & \text { Chaparro, D. } & \text { Investigación } \\ \text { Colombia } & \text { Acción }\end{array}$
familia de una relacionada, encuestas (a donde las problemáticas sociales, están Institución estudiantes, padres
familia). de afectando el ambiente escolar.

educación

secundaria.

Investigación

basada en la

Coquimbo, reconstrucción de

Chile, la

Retuert, G., y teorías subjetivas de

Serena

Castro, P. (2017). profesionales (Flick, 2009). En particular 30 docentes de 4 escuelas.

Análisis de contenido con procedimientos

Se explican las conductas al interior del aula recolección basados en los planteamientos de grounded theory (Glaser y Strauss, 1967). Con este propósito se aplican 4 entrevistas un grupo de grupales.

y orientan sus procedimientos para el manejo de la disciplina. A partir de esto, se establecen dinámicas de convivencia escolar que se reconstruyen continuamente y validan a través de los años de experiencia profesional.

docentes 


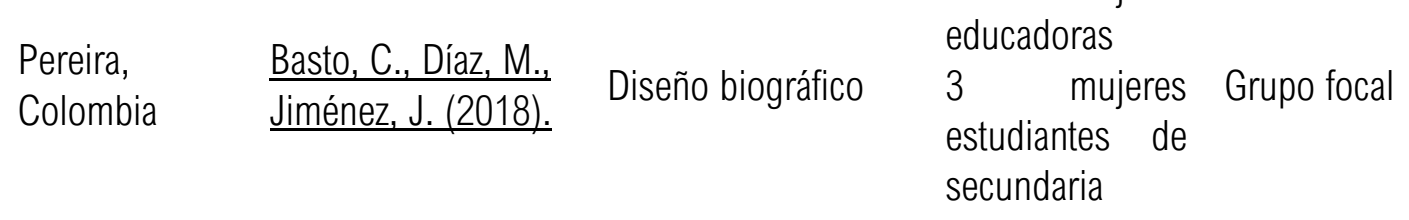

Ciudad Real, $\quad$ Carpio, C., Tejero,

España, la $\quad$ J., y García, V. Descriptiva

Mancha (2013).

$\begin{array}{lll} & & \text { Diseño } \\ \text { Madrid, } & \text { Carpio, C., y Tejero, } & \text { cuasiexperimental } \\ \text { España } & \text { J (2012). } & \text { con grupo control } \\ & & \text { no equivalente }\end{array}$

Pulido, R.,

Madrid, Calderón, S., Martín, G., Lucas, B. único (2014).
Se aplican pruebas para

89 alumnos de valorar el clima escolar y secundaria con cuestionarios de convivencia problemas de y las relaciones convivencia. interpersonales (docentes y alumnos).

Las técnicas estadísticas utilizadas fueron las de contraste de medias $y$ 89 alumnos/as análisis de varianza de 11 a 15 año multivariante. El análisis de datos se llevó a cabo con el programa SPSS (versión 14.0)

8 profesores orientadores y Observaciones y grupos de 10 estudiantes
El proceso de conciliación dentro de las instituciones educativas permite conseguir la armonía en la escuela. Se propone compartir espacios de sana convivencia mediante el dialogo, denotando habilidades: la empatía, la resiliencia, la práctica de valores, el perdón y la auto conciliación.

El abandono de los estudiantes puede generar una actitud negativa hacia la vida académica. Es necesaria la intervención del departamento de orientación para coordinar a los agentes educativos.

Es eficaz el trabajo preventivo de la violencia para mejorar la convivencia escolar, siendo recomendable ampliar el tiempo de intervención, los agentes implicados y los contextos familiar y social.

Se hizo un programa de mediación basado en diálogo, que facilitó una mejor convivencia en la escuela. Se señala la necesidad de contextualizar estos proyectos en una perspectiva a largo plazo. 


\section{Mérida, \\ Venezuela \\ Vera, A., y Lanz, R. (2017). \\ Descriptiva \\ 39 profesores \\ Encuesta}

39 profesores

Córdoba, Uruñuela, $P$.

España (2012)

Analítica descriptiva

de secundaria

CEP

Se realiza un análisis detallado de los programas

Córdoba.

de

de convivencia escolar.

$\begin{array}{lll}\text { Revisión } & 38 \quad \text { artículos } & \\ \text { bibliográfica de tipo } & \text { sobre } & \text { Bases de datos } \\ \text { descriptiva } & \text { convivencia } & \\ & \text { escolar } & \end{array}$

$\begin{array}{lll}\text { Maule, Chile Andrades, J. (2020). } & \begin{array}{l}\text { bibliográfica de tipo } \\ \text { descriptiva }\end{array} & \begin{array}{l}\text { sobre } \\ \text { convivencia } \\ \text { escolar }\end{array}\end{array} \quad$ Bases de datos

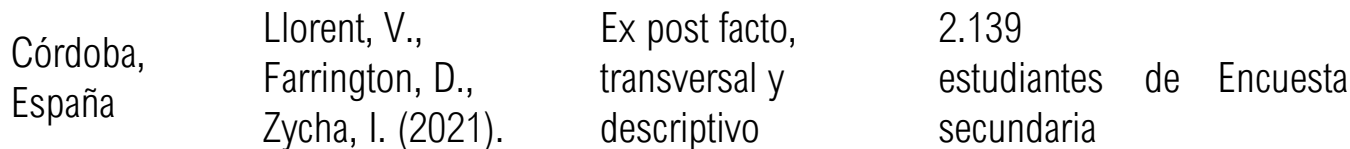

La poca organización, control, ejecución y evaluación de actividades escolares; escasa interacción escolar son resultado de la ausencia de buen clima escolar.

Los programas incluyen nuevos temas $y$, más allá de centrarse en la eliminación 0 disminución de la violencia, comienzan a proponer otras actividades enfocadas al desarrollo de las relaciones interpersonales, de las habilidades socioemocionales y de la vinculación de todo ello con el currículo de los centros. Se propone un plan de convivencia de dos tipos: reactivo 0 en positivo.

Factores que influyen en la convivencia escolar. Tales como: problemas con los resultados de aprendizaje, conflictos al interior del aula de clase, malas prácticas docentes, inclusión, violencia escolar.

Promover un clima escolar positivo a partir del plan de convivencia es conveniente, ya que la agresión del acoso escolar podría reducirse. Los hallazgos de este estudio tienen implicaciones en la política escolar y en las reformas educativas. 
385

Valdés-Cuervo,

Ciudad A.A., Martínez-

Obregón, $\quad$ Ferrer, B., y Carlos- Analítico

México Martínez, E.A.

(2018). adolescentes,

de 17 escuelas

secundarias de

un estado de

México, y 308

madres y 77

padres
Las prácticas docentes no permisivas y aquellas que implican a familias en la Modelo de ecuaciones prevención de la violencia se relacionan con estructurales con el su efecto en el clima escolar y la empatía. programa AMOS. Se concluye que estas prácticas previenen la violencia entre iguales, mejoran el clima escolar y la empatía. 


\section{DISCUSIÓN}

En respuesta a las preguntas iniciales y al objetivo de la revisión, los autores proponen la creación de programas basados en el diálogo y la reflexión docente (Carpio de los pinos y Tejero, 2012; García et al. 2016; Nail et al. 2013). También se proponen programas de mediación basados en el diálogo, en la formación de valores y los planes de convivencia (Uruñuela, 2012; Llorent et al. 2021; Basto et al., 2018; Cabrales et al. 2017; Pulido et al. 2014; Chaparro, 2019). Así como los basados en procesos conciliatorios y la construcción de normas dentro del aula de clases que mejoren la convivencia escolar (García et al. 2016; Basto et al. 2018; Nail et al. 2013).

En los programas de intervención para la convivencia escolar se destaca la labor del docente orientador (Delgado, 2018; Penalva et al. 2013; Carpio de los pinos y Tejero, 2013). Así como la interacción familia-escuela (Roncancio et al. 2017; Macías, 2017). Y finalmente se mencionan los factores que influyen en la convivencia y clima escolar (Chaparro, 2019; Andrades-Moya, 2020; Penalva et al. 2013; Vera y Lanz, 2017; Retuert y Castro, 2017; Carpio de los pinos et al. 2013; Valdés-Cuervo et al. 2018):

\section{Programas de intervención para la convivencia escolar}

Los programas de intervención para la convivencia escolar más allá de centrarse en la eliminación o disminución de la violencia, comienzan a proponer otras actividades enfocadas al desarrollo de las relaciones interpersonales, de las habilidades socioemocionales y de la vinculación de todo ello con el currículo de los centros (Uruñuela, 2012). Sin embargo, es necesario afrontarla desde la escuela con la promoción de un clima escolar armónico (Llorent et al. 2021). En este sentido, Martínez (2018) también propone un plan de formación en convivencia que apoye la transformación de conductas agresivas en posibilidades de aprendizaje a través de un programa de mediación escolar y proyectos pedagógicos.

Basto et al. (2018) mencionan entre las alternativas de intervención propuestas: la tutoría individual y de grupo. Los procesos mediadores como dos recursos viables que facilitan la convivencia y mejoran la convivencia escolar, así como un mejor conocimiento del lenguaje no verbal explicitado por el docente en el aula. En este sentido, la pedagogía social es la alternativa para el proceso de transformación, que a su vez contribuye a la generación de una cultura de paz (Cabrales et al. 2017). Es decir, se pone énfasis en programas de convivencia escolar que tengan como base la autonomía, el respeto, el diálogo y la solidaridad. Por lo antes expuesto, un aula de clase debe considerarse como un espacio en donde se reconozca y respete la diversidad, promoviendo la convivencia escolar que posibilite conocer a los otros más allá de lo institucional. En otras palabras, se estaría hablando de una educación que practique la democracia, que genere oportunidades para crear nuevas formas 
de relacionarse con la demás basada en valores tales como: justicia, libertad, autonomía y el diálogo (Basto et al. 2018).

Chaparro (2019) también propone el diálogo, la adecuada comunicación, y la formación en valores como intervención ante las dificultades de la convivencia escolar. Para ello, se requiere de la capacidad del docente, al hacer del acto educativo un espacio reflexivo, armónico con sentido holístico, para aprender desde el saber a construir un mundo más humano. Asimismo, Nail et al. (2013) consideran que el diálogo y la participación de los actores enfocado en la convivencia de aula ayuda a prevenir y construir la normativa escolar. Ante estos hechos se propone una estrategia de reflexión participativa fundamentada en la necesidad de la construcción colectiva entre los profesores de las normas para la vida en común en el aula; y la importancia atribuida al equipo directivo.

Otro programa de intervención para la convivencia escolar son los sistemas de apoyo que proporcionan una visión compartida de los problemas abriendo vías para iniciar diálogos en las escuelas. Como consecuencia, mejora el ambiente y clima de los centros (Jiménez, 2017). Se trata de un apoyo individualizado que se desarrolla en espacios diferenciados y categóricos lo que conlleva a hablar de segmentación y diferenciación de los alumnos para dar respuesta a sus necesidades. Consiste en desarrollar la educación inclusiva para disminuir la violencia. Por su parte, Carpio y Tejero (2012) consideran que es eficaz el trabajo preventivo de la violencia escolar para mejorar la convivencia escolar, por lo que se debe implicar a la familia en el contexto educativo.

Roncancio et al. (2017) también mencionan la importancia de trabajar en el proceso de interacción escuela y familia. Esta interacción permite comprender las habilidades emocionales de los estudiantes, por lo tanto, se espera que proporcione elementos para orientar la construcción de propuestas educativas que aborden con sentido pedagógico la alfabetización emocional.

Ahora bien, Carpio (2012) se enfoca en los adolescentes que ya han sufrido consecuencias de la violencia escolar, en aspectos específicos, como actitudes o comportamientos determinados, para proponer la construcción de programas que destaquen:

- Prevenir con grupos de riesgo

- Formación del profesorado para dotarles de estrategias de detección e intervención, se señala como prioridad.

- Enseñar a todos los alumnos a resolver conflictos y a pedir ayuda en caso necesario.

- Mejorar la relación entre los compañeros mediante metodologías que aporten experiencias positivas, como el aprendizaje cooperativo, o la discusión en grupos heterogéneos. 
- Romper la conspiración del silencio que está manteniendo estas situaciones, por parte de los compañeros, lo que es considerado por los agresores como apoyo implícito.

- Rebatir justificaciones de la violencia como "herramienta" para solucionar cosas, y desarrollar actitudes de tolerancia ante la diversidad.

- Colaborar desde la escuela y la familia y dar alternativas de ocio constructivo.

Por su parte, Pulido et al. (2014) mencionan un Programa de Mediación (para desarrollar una mejor convivencia en su escuela o el desarrollo de habilidades personales en estudiantes de cualquier escuela) y señala la necesidad de contextualizar estos proyectos en una perspectiva a largo plazo. Parece un factor clave para poder priorizar los objetivos a corto plazo, y considerar al menos un periodo de dos años para una implementación exitosa del programa y continuar con el proceso a pesar de las dificultades. Martínez (2018) propone un plan de formación en convivencia que apoye la transformación de conductas agresivas en posibilidades de aprendizaje a través de un programa de mediación escolar y proyectos pedagógicos. Los objetivos están orientados a determinar la modalidad, causalidad y frecuencia de los conflictos y a diseñar las estrategias que permitan canalizarlos e incluir a todos los miembros de la comunidad educativa en el programa de mediación y formación. En esta misma línea, Penalva et al. (2013) destaca su programa "Convivir es vivir", basado en valores de convivencia y una educación preventiva de conductas antisociales. Estos programas son desarrollados por estudiantes y profesores de las escuelas afectadas.

Por otro lado, Delgado (2018) destaca el afecto: donde está inmerso el diálogo, la escucha y el acompañamiento. Mientras que, Basto et al. (2018) hacen énfasis en el proceso de conciliación dentro de las instituciones educativas, el cual permite conseguir la armonía en las aulas de clases. Se denotan habilidades que enriquecen la vida del ser humano, como lo son: la empatía, la resiliencia, la práctica de valores, el perdón y la auto conciliación.

Finalmente, Valdés et al. (2018) consideran que las prácticas docentes no permisivas y aquellas que implican a familias en la prevención de la violencia se relacionan con una menor agresión entre pares, a través de su efecto en el clima escolar y la empatía. Se concluye que estas prácticas previenen la violencia entre iguales, mejoran el clima escolar y la empatía.

De esta manera, los programas de intervención para la convivencia escolar destacan el diálogo y la buena comunicación como base de un buen diseño y hacen énfasis en el rol del docente aunado a la familia, es decir, en la interacción familia y escuela. En ese sentido, se reafirma la construcción de programas basados en el diálogo reconsiderado las normas del plan de convivencia para la mejora del clima escolar y la reducción la violencia en los estudiantes. No obstante, se sigue dejando de lado la formación del docente orientador, es 
decir, los programas también deben considerar la formación para los docentes. Los autores no solo destacan el papel del docente orientador en el marco de la convivencia escolar, sino también el rol de la familia en la interacción familia-escuela (Delgado, 2018; Penalva et al. 2013; Carpio y Tejero, 2013, García et al., 2016; García, 2017; Valdés et al., 2018).

\section{Labor del docente orientador para abordar esta problemática}

Como se mencionó ut supra, al dar respuesta a la violencia escolar, se proponen la creación de programas de intervención para la convivencia, en los cuales el docente tiene un rol importante (Uruñuela, 2012). Partiendo del rol docente como mediador y gestor de la convivencia, se destaca la necesidad de promover los programas de formación para los docentes. Por lo tanto, los autores (Carpio, 2012; Carpio et al., 2013; Penalva et al., 2013;) enfatizan en estrategias de intervención, orientadas al desarrollo de actividades a nivel de aula y de centro, así como el trabajo curricular mediante el aprendizaje cooperativos donde se fomente el trabajo en grupo (Macías, 2017).

Los docentes orientadores, son aquellos que han pasado de ser instructores a tutores (Resolución $N^{\circ} 0008$ de 2020). Es preciso mencionar que el rol del docente orientador no sólo está direccionado a la intervención sino también a la resolución de conflictos y procesos conciliatorios escolares (Delgado, 2018). Al respecto, Macias (2017) menciona que los docentes orientadores, permiten orientar su enseñanza para mejorar el aprendizaje y el clima de convivencia, modificando su discurso didáctico y utilizando recursos semióticos cuya finalidad varía en función del momento en que aparecen. Estas actuaciones que los profesores emplean en el aula es un requisito para alcanzar la buena convivencia y así aumentar la efectividad de los procesos de enseñanza y aprendizaje. Así mismo, frente al rol del docente orientador en el marco de la convivencia, se destacan aspectos como la prevención, la promoción, intervención, remisión de casos, acompañamiento, conciliación direccionada en la resolución de conflictos, asesoramiento y detección de necesidades educativas especiales (Delgado, 2018).

Por su parte, Carpio et al. (2013) consideran que, los dos principales elementos del clima escolar son la calidad de la relación profesor-alumno y la calidad de la interacción entre compañeros, es decir, la convivencia escolar es un proceso de interacción entre el profesor, los alumnos y la familia. Como se mencionó inicialmente, mientras que, para mejorar la convivencia escolar, se necesitan programas de intervención; para los profesores orientadores se necesitan programas de formación que se adapten a sus necesidades pedagógicas y que desde las instituciones mejoren sus competencias en la gestión de la convivencia (Penalva et al., 2013). De hecho, Carpio (2012) respecto a los programas de mejora de la convivencia, propone la formación permanente de los profesores en innovaciones que implican cambios importantes en su papel docente. 
Con relación a lo anterior, se hace necesario, por lo tanto, promover la reflexión del docente tanto sobre sus propios valores como sobre aquellos que transmite a sus alumnos, así como facilitar la formación del profesorado respecto a todas aquellas cuestiones que guardan relación con la enseñanza de valores y su incidencia en la resolución de conflictos (García et al. 2016). Teniendo en cuenta la función del docente orientador, las investigaciones pudieran ser muy escasas o siempre están direccionadas al contexto educativo, pero el rol del docente orientador pareciera ser nulo. Resulta paradójico observar que en Colombia la figura del docente orientador parece nueva, es decir, el docente orientador apenas está incursionado por organizar, sistematizar, y vincularse a las problemáticas de la escuela.

\section{Factores asociados a la convivencia escolar}

Los factores asociados a la convivencia escolar son considerados un tema investigado por la necesidad de encontrar respuestas ante las situaciones de violencia escolar. Según Penalva et al. (2013), los factores escolares son principalmente los que causan problemas en la convivencia escolar. No obstante, Andrades (2020) considera que son los resultados de aprendizaje, los conflictos familiares, las malas prácticas docentes, los problemas con la inclusión educativa y la violencia escolar; los principales factores que afectan la convivencia. Por su parte, Vera y Lanz (2017) mencionan que la poca organización, control, ejecución y evaluación de actividades escolares; y la escasa interacción escolar son resultado de la ausencia de buen clima escolar.

Retuert y Castro (2017) destacan que la convivencia escolar está influida permanentemente por una serie de factores, que pueden asociarse a tres ámbitos de influencia recíproca: "Institucional”, el "Alumno" y el "Profesor". En este sentido, la percepción de los profesores hacia la gestión escolar; la percepción de los alumnos hacia la escuela y los factores relacionados con lo institucional, influyen en el tipo de convivencia escolar. Por otro lado, Carpio et al. (2013) describen los factores influyentes en la convivencia escolar como las agresiones verbales y físicas, la desobediencia de normas, la percepción negativa hacia la escuela, y otras conductas disruptivas son factores que dificultan la interacción escolar, el cultivo de un ambiente de paz en la vida cotidiana de los centros educativos, además de complicar la consecución de los objetivos de aprendizaje.

El contexto, también es un factor influyente en la convivencia escolar (Delgado, 2018), además de ser un componente esencial en la convivencia, en la orientación y en la construcción de las representaciones sociales, genera una acción contundente con la convivencia. Que van de la mano con el desarrollo moral de los estudiantes (Reyes et al., 2021). Es por esto, que la orientación escolar da la apertura al establecimiento de vínculos y lazos de comunicación entre la institución educativa y los intereses de los estudiantes. Dichos vínculos permiten permear las emociones, la autoestima, el autoconocimiento y las habilidades al lograr identificar las expectativas, necesidades y problemáticas de los integrantes de la comunidad educativa, con el 
fin de llegar a intervenciones efectivas para fortalecer la convivencia y la orientación escolar. Por su parte, Carpio (2012) también hace mención del contexto en el que se hace eficaz el trabajo preventivo de la violencia escolar para mejorar la convivencia, siendo recomendable ampliar el tiempo de intervención, los agentes implicados y los contextos familiar y social. Luego de hacer esta reflexión se encuentran puntos coincidentes con respecto a: la interacción profesor, alumno y escuela, destacando el contexto escolar, en el que la convivencia escolar parece estar influida por la interacción de los agentes implicados en la escuela. Como menciona, Arán (2021) es necesario implementar y desarrollar una cultura inclusiva que erradique las prácticas discriminatorias.

Dentro de las limitaciones de este estudio, se destaca principalmente el número de registros encontrados con las combinaciones de términos en los tesauros. Son pocos los estudios sobre los programas de intervención de convivencia escolar en población adolescente o básica secundaria. 19 artículos es poco para un tema de corte educativo que ha despertado interés desde la antigüedad. Además, el término "programas de intervención" suele aparecer en la búsqueda, pero no en todos los casos, está relacionado con programas para mejorar la convivencia escolar y reducir la violencia. Sin embargo, algunos autores, mencionan la importancia de promover estos programas, pero siguen siendo escasos.

No se puede desconocer que, este tema ha sido investigado desde hace décadas, pero en el estudio se consideraron como criterios de inclusión únicamente los últimos diez años para mostrar el estado actual de la investigación, por lo que el tiempo es considerado otra limitación. Por otro lado, en cada una de las investigaciones se queda corto el tema sobre "la intervención psicosocial", no se encontraron investigaciones asociadas a la convivencia escolar en estudiantes de secundaria. Y en todos los documentos, aparece el término "docente orientador" aunado al papel de la familia dentro de la escuela. Puesto que, en palabras de Toledo (2021) al implementar un modelo de orientación psicoeducativa no solo desarrolla una estrategia de acompañamiento y desarrollo de habilidades de aprendizaje metacognitivo, sino aspectos actitudinales y psicosociales de los actores inmersos en la educación.

\section{CONCLUSIONES}

En el abordaje de la convivencia escolar se destaca la participación del contexto (la escuela), de los docentes, de los alumnos y, por último, de la familia, quienes configuran un entramado de interrelaciones cuya influencia se evidencia en el clima escolar dentro de las aulas de clases. Dicho esto, se ha creado la necesidad de promover diferentes programas de intervención para la convivencia escolar; y programas de formación para los docentes orientadores. 
Los estudios encontrados hacen énfasis en la necesidad de seguir promoviendo la construcción programas de intervención y formación escolar, basados en los programas de convivencia, los cuales se fundamentan en el diálogo, la conciliación, la orientación escolar, la mediación, los grupos de apoyo y los planes de convivencia. Estos últimos, podrían ser reconsiderados basándose en el manual de convivencia y la construcción de normas desde la perspectiva de cada uno de los agentes del contexto escolar: el estudiante, el docente, la familia y la escuela.

Los autores apuestan por la necesidad de crear un modelo nuevo de convivencia que supere los retos de la educación actual. Este nuevo modelo de convivencia, podría enfocarse en la formación para los estudiantes y los docentes orientadores, basados en la reconstrucción del manual de convivencia. Asimismo, se propone brindar atención a casos particulares de violencia escolar, en los que se encuentre inmerso el docente orientador y el estudiante. Además, con la promoción de estos programas se identifican los elementos que se deben tener presente para la atención psicosocial de los docentes orientadores. De esta manera, se propone un plan de formación en convivencia que apoye la transformación de la violencia escolar y de climas escolares agresivos en posibilidades de aprendizaje e interacción a través de la resignificación del proyecto pedagógico y las actividades escolares.

\section{Conflicto de intereses / Competing interests:}

Los autores declaran que no incurre en conflictos de intereses.

\section{Rol de los autores / Authors Roles:}

Sunilda Soto: conceptualización, curación de datos, análisis formal, adquisición de fondos, investigación, metodología, administración del proyecto, recursos, software, supervisión, validación, visualización, escritura preparación del borrador original, escritura - revisar \& amp; edición.

Lizeth Reyes: conceptualización, análisis formal, investigación, metodología, administración del proyecto, recursos, software, supervisión, validación, visualización, escritura - preparación del borrador original, escritura - revisar \& amp; edición.

\section{Fuentes de financiamiento / Funding:}

Los autores declaran que no recibió un fondo específico para esta investigación.

\section{Aspectos éticos / legales; Ethics / legals:}

Los autores declaran no haber incurrido en aspectos antiéticos, ni haber omitido aspectos legales en la realización de la investigación.

\section{REFERENCIAS}

Amate, R., Ruiz, M. Inmaculada, Pérez, M., Carrión, J., y Gázquez, J. (2009). La convivencia escolar desde una perspectiva docente. International Journal of Developmental and Educational Psychology, 1(1),287-292. https://www.redalyc.org/articulo.oa?id=349832320031 
Andrades, J. (2020). Convivencia Escolar en Latinoamérica. Una revisión bibliográfica. Educare, 24(2). https://www.redalyc.org/journal/1941/194163269017/

Arán, A. (2021). La inclusión educativa en una escuela normal rural: un estudio de caso. Revista Estudios Psicológicos, 1(4), 7-32. https://doi.org/10.35622/j.rep.2021.04.001

Basto, C., Díaz, M., Jiménez, J. (2018). Vivencias en conciliación escolar de estudiantes y docentes mediadoras en la ciudad de Pereira. Plumilla Educativa, 21 (1). 99-119. https://dialnet.unirioja.es/servlet/articulo?codigo=6719759

Benítez, F. (2020). Las políticas de convivencia escolar colombianas en el contexto de América Latina. Revista Historia de la Educación Colombiana, 24 (24), 1-1. https://dialnet.unirioja.es/servlet/articulo?codigo=7788885

Cabrales, L., Contreras, N., González, L., y Rodríguez, Y. (2017). Problemáticas de convivencia escolar en las instituciones educativas del caribe colombiano: análisis desde la pedagogía social para la cultura de paz. Universidad del Norte, Barranquilla. http://manglar.uninorte.edu.co/bitstream/handle/10584/7693/130308.pdf?sequence=1

Carpio, C., y Tejero, J (2012). Eficacia de un programa sobre la prevención de la violencia en un centro de enseñanza secundaria. Revista de Española de Orientación y Psicopedagogía, 23 (2). https://www.redalyc.org/pdf/3382/338230791009.pdf

Carpio, C., Tejero, J., y García, V. (2013). Analysis of influential factors in a school environment with violence and coexistence difficulties | [Análisis de los factores influyentes en un ambiente escolar con violencia y dificultades de convivencia]. Revista Espanola de Orientacion y psicopedagogía, 24(3),124-134. https://doi.org/10.5944/reop.vol.24.num.3.2013.11249

Chaparro, D. (2019). Educar para la Sana Convivencia. Educación Y Ciencia, (23), 207-218. https://revistas.uptc.edu.co/index.php/educacion_y_ciencia/article/view/10232

De Benito, M., Elices, J., Francia, M., García, B., y Monjas, M. (2009). Cómo promover la convivencia. Programa de Asertividad y Habilidades Sociales (PAHS): (Educación Infantil, Primaria y Secundaria). Editorial: CEPE Ciencias de la Educación Preescolar y Especial. https://dialnet.unirioja.es/servlet/libro?codigo=573133

Delgado, A, (2018). Orientación escolar en el marco de la convivencia escolar una mirada desde las representaciones sociales. Revista Electrónica en Educación y Pedagogía, 12 (2),100-112. https://doi.org/10.15658/rev.electron.educ.pedagog18.03020208 
García, M. Sola, J., y Peiró, S. (2016). Los docentes como clave en la construcción de la convivencia escolar. Edetania: estudios y propuestas socio-educativas, 50. 69-83. https://dialnet.unirioja.es/servlet/articulo?codigo=6039914

García, M. (2017). Despliegue de la subjetividad docente: Percepciones y proyecciones axiológicas respecto a la convivencia escolar. Tesis doctoral. Universidad de Alicante. https://dialnet.unirioja.es/servlet/tesis?codigo=121082

Jiménez, A. (2017). La creación, formación y desarrollo de grupos de apoyo mutuo entre estudiantes para la mejora escolar y social. Tesis doctoral. Universidad de Sevilla. https://dialnet.unirioja.es/servlet/tesis?codigo=121179

Llorent, V., Farrington, D., Zycha, I. (2021). El plan de convivencia y su relación con las competencias socioemocionales, el bullying y el cyberbullying en la educación secundaria. Revista de Psicodidáctica, 26 (1), 35-44. https://doi.org/10.1016/j.psicod.2020.11.002

Macias, E. (2017). Interacción comunicativa y la convivencia escolar en el aula de educación secundaria. Tesis doctoral. Universidad de Extremadura. https://dialnet.unirioja.es/servlet/tesis?codigo=107782

Martínez, M. (2018). La formación en convivencia, papel de la mediación en la solución de conflictos. Educación y Humanismo, 20 (35), 129-144. https://dialnet.unirioja.es/servlet/articulo?codigo=6510625

Moher, D., Liberati, A., Tetzlaff, J., Altman, D. G., y Prisma Group. (2009). Preferred Reporting Items for Systematic Reviews and Meta-Analyses: The PRISMA Statement. Annals of Internal Medicine, 151(4), 264-269. https://doi.org/10.1371/journal.pmed.1000097

Nail, 0., Muñoz, M., y Gajardo, J. (2013). Principios orientadores de la convivencia en el aula: una estrategia de reflexión colectiva. Educaçao e Pesquisa: Revista da Faculdade de Educação da Universidade de São Paulo, 39 (2), 367-386. https://dialnet.unirioja.es/servlet/articulo?codigo=4503058

Penalva, A., Hernández, M., Guerrero, C. (2013). ¿La formación del profesorado para la mejora de la convivencia escolar según la percepción de expertos? Revista fuentes, (15), 281-304. http://dx.doi.org/10.12795/revistafuentes.2014.i15.13

Pulido, R., Calderón, S., Martín, G., Lucas, B. (2014). Implementation of a school mediation program: Analyzing the perceived difficulties and ways to improve it | [Implementación de un programa de mediación escolar: Análisis de las dificultades percibidas y propuestas de mejora]. Revista Complutense de Educación, 25(2), 375-392. 10.5209/rev_RCED.2014.v25.n2.41610

Retuert, G., y Castro, P. (2017). Teorías subjetivas de profesores acerca de su rol en la construcción de la convivencia escolar. Polis: Revista Latinoamericana, 16(46). https://dialnet.unirioja.es/servlet/articulo?codigo=6077437 
Reyes, L., Núñez, A., Núñez, A., Sánchez, M., Carmona Alvarado, F. A., Raphael Bataglia, P. U., \& Paiva Alves, C. (2021). Desarrollo moral en contextos educativos: Una revisión sistemática. European Journal of Child Development, Education and Psychopathology, 9(1), 1-32. https://doi.org/10.32457/ejpad.v9i1.1508Rodríguez, J. (2008). Los docentes ante las situaciones de violencia escolar. Revista electrónica interuniversitaria de formación del profesorado, 11 (3). https://dialnet.unirioja.es/servlet/articulo?codigo=2782156

Roncancio, M., Camacho, N., Constanza, J., Vaca, P. (2017). Convivencia escolar y cotidianidad: una mirada desde la inteligencia emocional. Educación y Desarrollo Social 11 (1), 24-47. https://dialnet.unirioja.es/servlet/articulo?codigo=6039564

Toledo, F. (2021). La orientación psicoeducativa en las instituciones de educación superior como estrategia de acompañamiento al aprendizaje. Revista Estudios Psicológicos, 1(4), 102-121. https://doi.org/10.35622/j.rep.2021.04.005

Uruñuela, P. (2012). La formación del profesorado en convivencia. Revista digital de educación y formación del profesorado, (9), 252-269. https://dialnet.unirioja.es/servlet/articulo?codigo=6423529

Valdés, A.A., Martínez, B., y Carlos, E.A. (2018). El rol de las prácticas docentes en la prevención de la violencia escolar entre pares. Revista de Psicodidáctica, 23 (1), 33-38. https://doi.org/10.1016/j.psicod.2017.05.006

Vera, A., y Lanz, R. (2017). La planificación como estrategia gerencial para el fortalecimiento de la convivencia escolar. Educere, 21 (68), 155-164. https://www.redalyc.org/journal/356/35652744014/

Yoneyama, S. y Rigby, K. (2006). Bully victim students and classroom climate. Youth StudiesAustralia, 25 (3), $34-41$.

https://www.researchgate.net/publication/286172927_Bullyvictim_students_and_classroom_climate 